\title{
A narrativa como estratégia na compreensão da experiência do adoecimento crônico: uma revisão de literatura
}

\author{
Narrative as a strategy for understanding the experience \\ of chronic illness: a literature review
}

Tania Gonçalves Vieira Caçador (https://orcid.org/0000-0002-1975-7148) ${ }^{1}$

Romeu Gomes (https://orcid.org/0000-0003-3100-8091) ${ }^{1}$

${ }^{1}$ Instituto Sírio-Libanês de Ensino e Pesquisa. R. Prof. Daher Cutait 69, Bela Vista. 01308-060 São Paulo SP Brasil.taniavieiracacador@ gmail.com

\begin{abstract}
This article aims to explore, in available literature, the use of narrative in studies of chronic illness. It is hoped that this study in addition to knowing how to have been the use of narrative in studies, contribute to the care of people with chronic illness. Methodologically, the study consists of a literature review of qualitative nature, focusing on articles with the Virtual Health Library descriptors (VHL). In terms of results, three stand out meanings attributed to the use of narrative by the authors: technique to get testimonies, research method and specific type of testimony; and in terms of the main results attributed by the authors with the use of narrative, are evident two themes: the experience of chronic illness in the subject ill perspective, highlighting the search for meaning, reframing the chronicity and coping strategies; and the possibility of understanding chronic illness, by professionals from the experience of the subject ill. It is verified, in the analyzed studies, that the use of the narrative itself or the narration process can make possible the resignification of the process of illness by the subjects and point out strategies for care management in chronic illness.
\end{abstract}

Key words Narration, Chronic disease, Methodology, Qualitative research, Life changing events
Resumo O artigo objetiva explorar, na literatura disponivel, o uso da narrativa em estudos sobre o adoecimento crônico. Espera-se com esse estudo além de conhecer como têm sido o uso da narrativa nos estudos, contribuir na atenção a pessoas com adoecimento crônico. Metodologicamente, o estudo consiste numa revisão bibliográfica de cunho qualitativo, focalizando artigos com descritores da Biblioteca Virtual em Saúde (BVS). Em termos de resultados, destacam-se três sentidos atribuídos ao uso da narrativa pelos autores: técnica para obter depoimentos, método de pesquisa e tipo específico de depoimento; e em termos de principais resultados atribuídos pelos autores com o uso da narrativa, evidenciam-se dois temas: a experiência do adoecimento crônico na perspectiva do sujeito adoecido, com destaque a busca de sentido, ressignificação da cronicidade e estratégias de enfrentamento; e a possibilidade de compreensão do adoecimento crônico, por parte dos profissionais, a partir da experiência do sujeito adoecido. Verifica-se, nos estudos analisados, que o uso da narrativa em si ou o processo de narrar pode possibilitar a ressignificação do processo de adoecimento pelos sujeitos e apontar estratégias para a gestão do cuidado no adoecimento crônico. Palavras-chave Narrativa, Doença crônica, Metodologia, Pesquisa qualitativa, Acontecimentos que mudam a vida 


\section{Introdução}

No cenário atual, no qual produções apontam para questões relativas a processos crônicos de adoecimento, caracterizados pela longa duração, cuidado mais contínuo e longitudinalidade da atenção, fazendo com que o curso e a duração das doenças para os sujeitos, além de seus impactos para o sistema de saúde, passem a ser mais focalizados do que a própria transmissão das doenças ${ }^{1}$. Dentre os diversos aspectos que devem ser repensados para se lidar com esse cenário, destacamos que o lugar da fala dos sujeitos acerca de suas experiências na temporalidade do adoecimento crônico afigura-se como de fundamental importância.

Nessa vertente, estudos narrativos têm sido incorporados nas últimas décadas na produção de conhecimento nas pesquisas qualitativas na área da saúde, principalmente na compreensão da experiência do sujeito adoecido.

Segundo Bury ${ }^{2}$ a narrativa de histórias, quer sobre si mesmo ou outros, é universal; e possibilita a articulação entre o corpo, o self e a sociedade.

Nessa mesma perspectiva, Castellanos ${ }^{3}$ considera que as narrativas mediam o "interior" e o "exterior" ao "eu" na relação ser-no-mundo. As narrativas então podem ser compreendidas como modos de elaboração da experiência social, descrevendo situações vivenciadas num determinado tempo.

$\mathrm{Good}^{4}$ considera que a narrativa é uma forma onde a experiência é representada e relatada, numa determinada sequência. $O$ relato da experiência, a temporalidade (desdobramento no tempo), a interação entre os personagens, o contexto "virtual" (cenário) e a trama (enredo) são elementos essenciais e caracterizam uma narrativa.

Compreende-se, neste trabalho, a narrativa como estratégia para representar e relatar a experiência, onde uma característica central é a temporalidade. No relato ocorre uma articulação entre passado, presente e futuro ${ }^{5}$.

Hyden $^{6}$ aponta que "uma das formas mais poderosas de expressar o sofrimento e as experiências relacionadas ao sofrimento é a narrativa. As narrativas dos pacientes dão voz ao sofrimento de um modo que está fora do domínio da voz biomédica"(p.49). Refere ainda ser esse um dos prováveis motivos para o interesse em narrativas entre os cientistas sociais em pesquisas no campo da biomedicina, doença e sofrimento.

Nesse sentido, as narrativas têm colaborado, nos últimos anos, na construção de novas abor- dagens sociológicas e antropológicas das enfermidades crônicas, uma vez que provoca ruptura na centralidade do modelo biomédico. Ao possibilitar voz aos pacientes, através das narrativas, estes se tornam capazes de ressignificar suas vidas, desta forma reduz a exclusividade da medicina na melhora do cuidado ${ }^{7}$.

Por cronicidade, Canesqui ${ }^{7}$ refere ser um conceito da sociedade ocidental e diz respeito "às condições de saúde que podem ser gerenciadas, mas não curadas, apresentando sintomas contínuos ou periódicos que, de certo modo, podem interferir em várias dimensões da vida do adoecido e no seu entorno"(p.20).

Bury $^{8}$, num estudo sobre narrativas de mulheres com artrite reumatoide, conceitua a doença crônica como um tipo de evento disruptivo, onde os sujeitos sofrem uma ruptura das estruturas da vida cotidiana e na forma de compreensão que as sustentam. Tal ruptura mobiliza nesse sujeito recursos de diversas ordens para lidar com a nova situação que afeta sua trajetória de vida, incluindo o rever sua biografia e autoimagem. Assinala que uma das características principais da doença crônica é a forma insidiosa que se apresenta na vida do sujeito.

Apesar da existência de críticas relevantes a respeito dos conceitos de reconstrução narrativa e ruptura biográfica concebidos nesse estudo $\left(\right.$ Bury $\left.^{8}\right)$, Castellanos ${ }^{9}$ defende o uso, já que estes "mostraram-se extremamente sensíveis e relevantes para a análise da experiência do adoecimento grave"(p.51), inclusive compreendendo ser utilizada mesmo na análise de doenças genéticas.

Para Canesqui ${ }^{7}$ esse resgate dos estudos das narrativas, não é um fato exclusivamente nacional, havendo uma tendência atual do uso da abordagem qualitativa da subjetividade em saúde e o adoecimento crônico, contrapondo a experiência subjetiva do sujeito e enfoque macroestrutural.

O estudo das narrativas do adoecimento crônico, tal como referido por Bury ${ }^{2}$, pode lançar luz sobre o significado da doença e da experiência interrompida, bem como ações/atitudes para lidar com a nova situação.

As narrativas dos sujeitos acometidos pela doença crônica podem trazer aspectos do reconhecimento da doença, alterações na sua vida, vivência da dor e do sofrimento, o vislumbre da incapacidade ao longo do tempo, a incerteza do curso da doença, a expectativa da cura e o significado do evento para o sujeito. A narrativa então é uma possibilidade para a reorganização dos re- 
cursos individuais podendo possibilitar que o sujeito a partir da vivência (passado), ressignifique o evento e projete o futuro nessa nova condição ${ }^{8}$.

Além dessas contribuições, a narrativa também pode ser associada com mudanças, da parte dos profissionais, para melhorar o cuidado. De acordo com Greenhalgh e Hurwitz ${ }^{10}$ "entender o contexto narrativo da doença fornece uma estrutura para abordar os problemas de um paciente de forma holística, bem como revelando opções de diagnóstico e terapêutica”(p.49).

Nessa perspectiva, define-se como objeto de estudo deste trabalho o uso da narrativa como estratégia de compreensão do processo saúdedoença-cuidado, tendo como foco o adoecimento crônico. Objetiva-se discutir as contribuições, limites e possibilidades do uso da narrativa no campo da saúde, a partir da exploração de literatura nacional e internacional, que utilizam narrativas de adoecimento crônico.

$\mathrm{Na}$ literatura internacional a narrativa tem sido utilizada como forma de melhor compreender a doença por inúmeros autores. Dentre eles, destacam-se Bury ${ }^{2}$, Good ${ }^{4}$ e Kleinman ${ }^{11}$ com seus estudos. Esses autores dedicam suas discussões na experiência da doença crônica. Devido ao longo prazo e mudanças que ocorrem nas histórias de vida dos sujeitos, a narrativa pode ser uma importante estratégia para melhor compreender a experiência do adoecimento.

Com base nessas considerações, definimos a seguinte questão para o nosso estudo: Como o uso da narrativa pode contribuir para uma melhor compreensão da experiência da doença crônica?

Face o exposto, justifica-se o presente estudo que pretende sistematizar o que foi produzido até o momento, conhecer como têm sido o uso da narrativa em estudos sobre o adoecimento crônico e também apontar novas perspectivas do uso da narrativa na atenção a pessoas com adoecimento crônico.

\section{Trajetória metodológica}

Este estudo é parte de uma pesquisa mais ampla que explora o uso da narrativa no âmbito da saúde, aprovada pelo Comitê de Ética do Hospital Sírio Libanês. Caracteriza-se como uma revisão da literatura numa abordagem qualitativa, compreendida como um estudo exploratório da produção do conhecimento acerca de um assunto ou tema. $\mathrm{O}$ estudo bibliográfico além de possibilitar o alcance de um amplo número de informações e dados dispersos em várias publicações, também auxilia na melhor definição do quadro conceitual que envolve o objeto de estudo proposto ${ }^{12}$.

A fim de compreender como têm sido o uso da narrativa na experiência do adoecimento crônico, empreendeu-se uma busca bibliográfica nas bases de dados disponíveis no Portal Regional da Biblioteca Virtual em Saúde (BVS) ${ }^{13}$, abrangendo todo o período da base da BVS, 1982 a 2017.

Para a elaboração da estratégia de busca foi necessário a combinação de várias palavras-chave, em campo livre, pois não há descritores disponíveis no vocabulário controlado de termos em saúde (Tesauro) da BVS, para o termo "adoecimento crônico".

Relativamente à pesquisa, foram utilizadas as seguintes palavras-chave: doença crônica, tendo como resultado 239.377 artigos. Com a inclusão do termo narrativa, obteve-se 137 artigos. De acordo com o objetivo da pesquisa, foram utilizados como critérios de inclusão artigos qualitativos sobre doença crônica que incluíam a narrativa em sua metodologia ou em sua discussão; e como critério de exclusão artigos sobre doença crônica de caráter epidemiológico, etiológico, prognóstico, revisão quantitativa, medicamentos, reabilitação e análise ou descrição exclusivamente quantitativa. Na Figura 1, ilustramos a estratégia de busca.

A aplicação dos critérios de inclusão e exclusão supracitados, conduziram à obtenção de um total de 51 artigos. Após a leitura na íntegra destes, foram excluídos 15 títulos, por apresentar o mesmo artigo em diferentes idiomas, análise de dados quantitativos e os demais porque apesar de constar nos descritores iniciais doença crônica e narrativa, não apresentavam esses termos em seu corpo. Assim, constituem-se como fonte dessa revisão 36 artigos.

O primeiro movimento analítico das fontes foi um tratamento descritivo, caracterizando a produção quanto ao país, ano de publicação, tema do adoecimento crônico e abordagem metodológica.

Diante da multiplicidade de desenhos metodológicos e até mesmo ausência de explicitação em alguns artigos, adotou-se as seguintes categorias: Pesquisa teórica (estudo conceitual ou de defesa de premissas com base teórica); Pesquisa de campo (estudo com a produção de fontes primárias em grupos ou conjunto de relações); Estudo de caso (estudo baseado na investigação de particularidades de fenômenos de um contexto de vida); Artigo Opinião (trabalho baseado numa opinião qualificada de autores); Pesquisa Docu- 


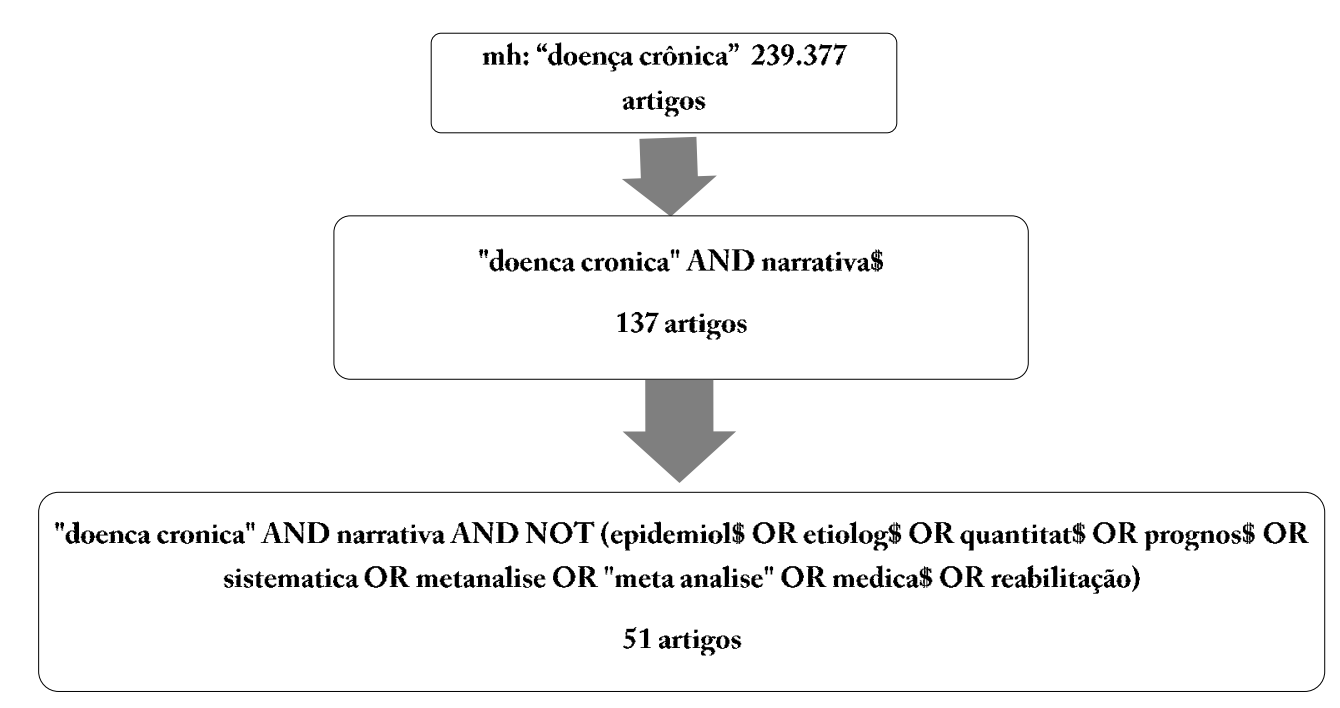

Figura 1. Estratégia de busca.

mh: Termo indexado no DeCS - Descritores em Ciências da Saúde; $\$$ = símbolo que permite a busca de uma palavra considerando qualquer sufixo.

mental (Estudo de fontes primárias que não tiveram tratamento analítico), Revisão da literatura (estudo de fontes secundárias); Relato de experiência (estudo baseado na descrição de uma experiência) e Editorial (opinião do corpo editorial de um periódico acerca de um assunto).

Em um segundo movimento analítico formulou-se questões a fim de problematizar a produção com ancoragem na conceituação de narrativa como uma forma onde a experiência é representada e recontada, dando possibilidade ao narrador perceber uma articulação entre presente, passado e futuro, tendo personagens, cenários e temporalidade 4 .

Em termos operacionais, a partir da questão inicial do estudo, foram formuladas subquestões e etapas, ilustradas na Figura 2.

\section{Caracterização das fontes}

No primeiro momento, na busca de um panorama do conjunto dos artigos localizados na revisão, observam-se diversas características dos estudos realizados que, esquematicamente, podem ser apontadas no Quadro 1.

Inicialmente, observa-se a escassez de produção nesse campo no Brasil, contabilizando apenas 3 estudos. Na literatura internacional há o predomínio de estudos norte-americanos, seguido dos europeus com evidência dos estudos britânicos. Isso não significa que não haja publicações fora desse cenário. Pode ser que haja produções que foram publicadas em periódicos que não atendam aos critérios de indexação da BVS.

Outro destaque ocorre quanto à produção relacionada ao descritor "doença crônica", que ultrapassa de 200.000 artigos. À medida que se delimita através do descritor narrativa e aplica-se os critérios de inclusão e exclusão, esse número reduz para metade de uma centena. Quanto ao ano de publicação, não se observa o predomínio em um determinado ano.

Em relação aos desenhos metodológicos empregados nos artigos, 22 utilizaram pesquisa de campo, 5 pesquisas teóricas, 4 estudos de caso, 1 artigo-opinião, 1 pesquisa documental, 1 revisão de literatura, 1 relato de experiência, 1 editorial. Este resultado aponta para uma predileção do uso de fontes primárias para abordar o adoecimento crônico e a perspectiva do sujeito adoecido.

No que se refere ao tema do adoecimento crônico relatados nos estudos, além de tratar de doença crônica e tipos específicos dessa doença, focaliza-se especificidades de faixas etárias (crianças, adolescentes, idosos) e o predomínio do gênero feminino. 

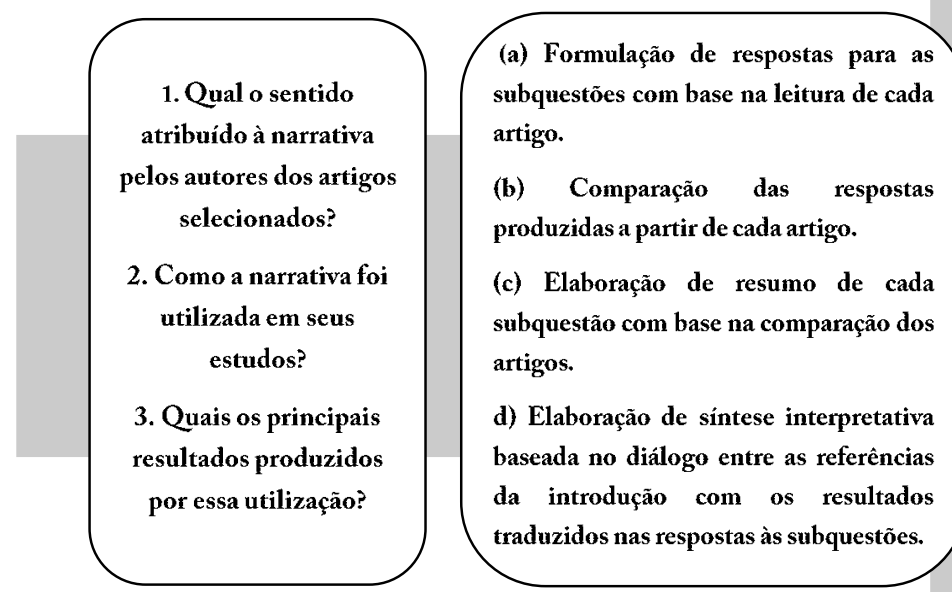

Figura 2. Subquestões e etapas.

Diante o número elevado dos estudos caracterizados como pesquisa de campo, considerouse relevante caracterizar quem eram os sujeitos da pesquisa e cenários onde estas foram realizadas. Os pesquisados foram predominantemente os próprios sujeitos do adoecimento e os cenários utilizados para a pesquisa na maior parte dos estudos não foram referidos.

\section{Sentidos atribuídos à narrativa em si}

Os autores, em geral, atribuem vários sentidos à narrativa como estratégia de pesquisa. No conjunto dos sentidos, observamos que tanto há uma convergência entre eles como uma intercomplementariedade. Um dos sentidos atribuídos à narrativa é a história de vida ${ }^{14-16}$. Associado a esse sentido, a temporalidade se destaca ${ }^{15,17-19}$. Assim, as histórias de vida são situadas num quadro temporal, que não só reconstitui o passado no presente, como também projeta o futuro por meio da ressignificação do passado. O contar a história pode assumir diferentes formas de ordenamento ou diferentes formas de encadear os fatos $^{15,17,20,21}$.

Outro sentido que se destaca é o relato de experiências ${ }^{14,15,17,19,22,23}$. Para os autores o relato dessas experiências não se limita a expressão de individualidades, mas também recobrem aspectos que nitidamente se afiguram como um diálogo entre indivíduo e cultura/sociedade ${ }^{14,19,23-25}$.
Nesse sentido, significados culturais servem de ancoragem para os relatos pessoais ou tais relatos são construídos por meio das relações societárias. Em outras palavras, os relatos refletem as relações estabelecidas entre o narrador e a audiência para qual se dirige a narrativa, ou ainda entre narrador e os personagens que emergem em suas narrativas. Além dos personagens, outros elementos fundamentais das narrativas são os enredos e cenários ${ }^{26,27}$.

A dimensão cultural também se expressa quando na narrativa são utilizadas metáforas ${ }^{22}$ que precisam de uma compreensão de significados culturais para que façam sentido. Há também o sentido da constituição do ser associado à narrativa ${ }^{18,20,28}$. À medida que a história pessoal é contada e ouvida, ao sentirem protagonistas, as pessoas afirmam e constroem a sua identidade.

Observamos também que há autores que não explicitam o conceito de narrativa ${ }^{28-38}$. Utilizam o termo como se já houvesse uma compreensão acerca do seu significado, e em alguns estudos, nos parece que está implícito nesse termo a produção de depoimentos em geral.

Por fim, destacamos que nos estudos analisados, há um autor que estabelece uma tipologia de narrativas, classificando-as em grandes e pequenas. Vivemos imersos em "grandes" e "pequenas" narrativas. As grandes nos situam em cosmologias e sistemas explicativos do mundo de ordem religiosa, científica, filosóficas, etc. Ao 
作

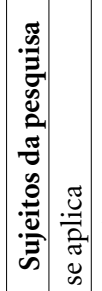

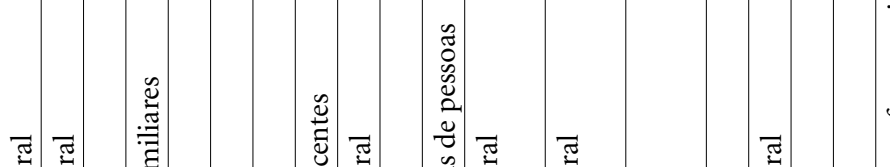

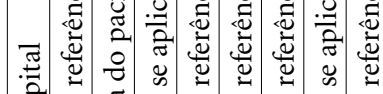

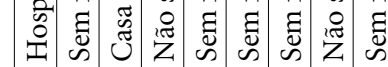

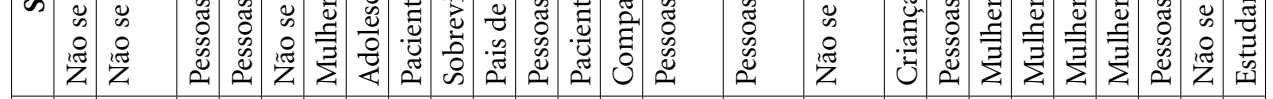

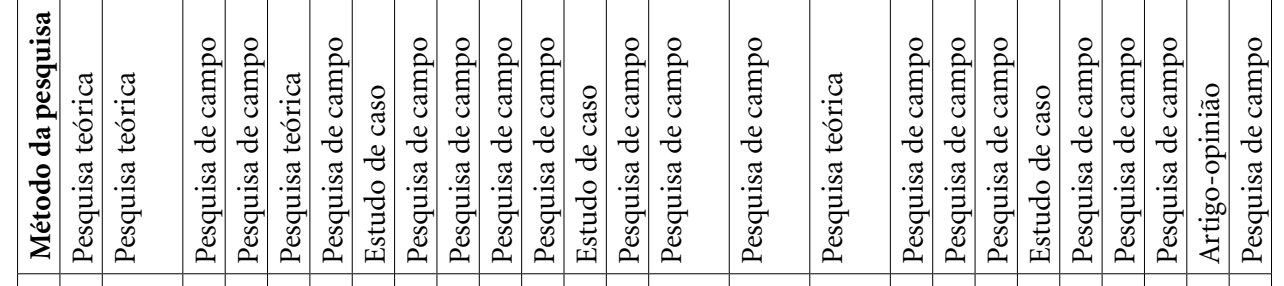

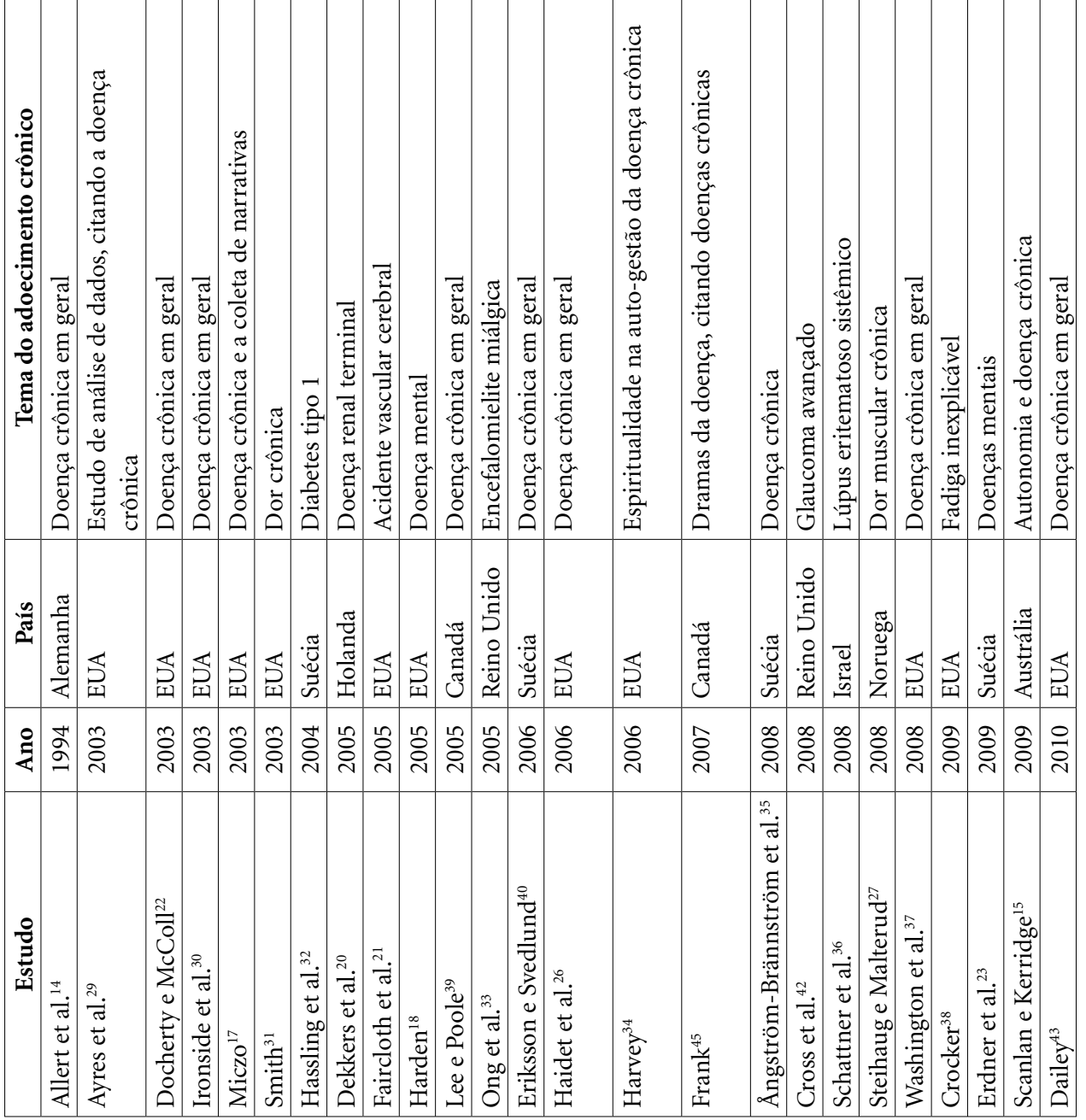




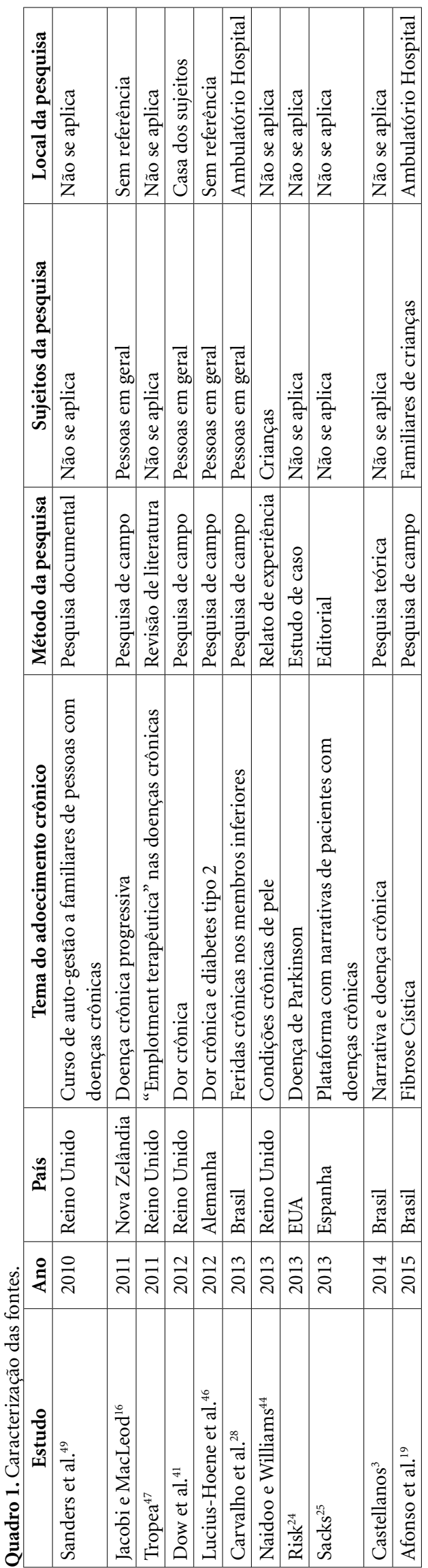

mesmo tempo em que ordenam eventos e ações em temporalidades mundanas ou sagradas, essas narrativas são reafirmadas ou transformadas através de ações e eventos que afetam tais temporalidades (revolução copérnica, iluminismo etc.). As pequenas narrativas estão presentes em diferentes contextos cotidianos, como família, escola, trabalho, consultório. Essas narrativas podem consistir em histórias curtas, produzidas em contextos interativos cotidianos; mas, também, podem orientar-se pela perspectiva biográfica. Em ambos os casos, podemos identificar instâncias de significação da relação eu/mundo ${ }^{3}$.

Em síntese, foram atribuídos às narrativas pelos autores os seguintes sentidos:

História de vida - Destaque a temporalidade;

Relato de experiências - Diálogo da Individualidade com cultura/sociedade e/ou com personagens, enredos e cenários;

Constituição do ser - Afirmação e construção da identidade; $\mathrm{e}$

Produção de depoimento - Depoimentos em geral.

\section{Sentidos atribuídos ao uso da narrativa}

Alguns autores caracterizaram o uso de narrativa em seus estudos como depoimentos a ser conseguidos por meio de entrevista "aberta", sem que houvesse perguntas ou roteiro prévios a serem seguidos por parte do entrevistador ${ }^{18,36,39-41}$. Nesse sentido, era fornecido ao entrevistado um estímulo para que esse desse o seu depoimento acerca de como vivia com a sua doença ou o que pensavam acerca dela. Ainda em termos de uso da narrativa como aplicação de entrevista, há um estudo que a denomina de "aprofundada" 21.

Nesse caso, não fica claro se esse aprofundamento se dá por conta da possibilidade de o entrevistado detalhar suas ideias ou pelo fato de o entrevistador ao longo da entrevista aprofundar o que era dito com perguntas para que houvesse um maior desenvolvimento das ideias contidas no depoimento. Por último, em termos de narrativa entendida como uma estratégia a ser obtida por meio de entrevista, há estudos que atribuem o uso como entrevistas semiestrutu$\operatorname{radas}^{16,18,20,22,26,27,34,42,41}$, dando a entender que não havia perguntas fechadas para se obter respostas dirigidas a perguntas. Num ou noutro sentido, a entrevista foi utilizada como uma técnica para se obter depoimentos.

Observou-se também que dois estudos ${ }^{19,26}$, não associaram a narrativa apenas à técnica a ser utilizada para produzir um determinado tipo 
de depoimento, mas a situaram na categoria de um método de pesquisa, utilizando elementos centrais de uma narrativa. Nessa acepção, não só enfatizaram a entrevista aberta como forma de se chegar a um depoimento, mas também apresentaram referências teóricas que fundamentam o trabalhar com narrativa, bem como princípios específicos para analisá-la.

Ainda no que se refere ao uso da expressão "narrativa", observa-se que, em geral, não é qualquer tipo de depoimento acerca da doença que se espera obter. Trata-se de depoimento que se afigura como a história de vida dos sujeitos da doença ou como menção e/ou caracterização de eventos associados ao viver com a doença ${ }^{16,20,30}$.

Outro sentido também associado ao termo em questão é a narrativa como um discurso da experiência ${ }^{18,22,28,41-43}$, sem que necessariamente se desconsidere a dimensão espaço-temporal.

E por fim, há estudos onde não está explícito o uso da narrativa, nos parece, como apontado no sentido atribuído a narrativa, está relacionado a uma fala ou a um depoimento em geral ${ }^{21,31,33,35,44}$.

Em resumo os sentidos atribuídos quanto ao uso da narrativa pelos autores foram:

Técnica para obter depoimentos;

Método de pesquisa; e

Tipo de depoimento esperado.

\section{Principais resultados atribuídos ao uso da narrativa}

Entre os resultados nota-se uma correlação e complementariedade no que diz respeito à captação da experiência do adoecimento crônico. Essa experiência é citada no sentido de desafios e atitudes ${ }^{20}$, no impacto dessa experiência na vida cotidiana $^{43}$ e na necessidade de dar um contexto para essa experiência ${ }^{14,29}$.

Nessa mesma perspectiva, alguns autores relacionam essa experiência com a busca de significado do adoecimento ${ }^{26,27,39,45}$. Vários autores referiram como resultado do uso da narrativa a produção de insights, a busca por novos caminhos e estratégias de enfrentamento ${ }^{16,22,38,39,46}$. Nesse sentido, um dos principais resultados do uso da narrativa é a possibilidade de o sujeito adoecido interpretar e ressignificar a sua cronicidade $16,21,22,24,27,37,39,45,47$.

Um outro grupo de autores apresentaram como principal resultado implicações no tratamento, na qualidade, no cuidado e no prognóstico ${ }^{15,19,21,26,36,40}$, nos parece que estes dados não seriam acessados considerando apenas o modelo biomédico. Desta forma, se torna importante para os profissionais compreenderem o adoecimento crônico.

Há um autor que aponta a necessidade de cautela da parte dos pesquisadores no uso da narrativa, uma vez que dando voz aos pacientes, há a possibilidade de manipulação do narrador através da narração seletiva de histórias ${ }^{17}$.

Em síntese, os principais resultados atribuídos ao uso da narrativa foram:

A experiência do adoecimento crônico - com destaque a busca de sentido, ressignificação da cronicidade e estratégias de enfrentamento; e

Compreensão do adoecimento na perspectiva do sujeito adoecido.

\section{Síntese Interpretativa}

De modo geral, podemos estabelecer um diálogo entre os sentidos atribuídos à narrativa analisados com o referencial conceitual que introduzimos neste trabalho.

O sentido de narrativa como história de vida é destacado no estudo de Bury ${ }^{2}$, onde afirma que "narrar histórias, sobre si ou sobre o outro, é um ato universal", que são formadas a partir da elaboração do uso da narrativa como forma de compreensão do processo saúde-doença-cuidado, tendo como foco o adoecimento crônico e uso de metáforas e outras figuras de expressão. Esse autor atenta também ao fato de que esse repertório simbólico é essencial para todas as culturas e segmentos culturais com a finalidade de obter uma integração social, assim como afirma que as narrativas das doenças crônicas têm papel importante na compreensão do tecido social e das contradições da interação social.

Good $^{4}$ também refere ser a narrativa o relato da história de vida e aponta que através da narrativa a experiência é representada e recontada, e os acontecimentos se apresentam numa ordem significativa e coerente, não somente informando, mas também descrevendo a experiência no passado, presente e a perspectiva para o futuro. Reconhece a temporalidade como elemento essencial da narrativa, assim como a interação entre os personagens, o cenário e o enredo. Nessa perspectiva, os autores Allert et al. ${ }^{14}$, $\mathrm{Miczo}^{17}$, Harden $^{18}$, Lee e Poole ${ }^{39}$, Haidet et al. ${ }^{26}$, Steihaug e Malterud ${ }^{27}$ e Afonso et al. ${ }^{19}$ atribuíram sentido alinhados ao referencial utilizado nesse estudo.

Há uma quantidade de autores que não explicitam o conceito de narrativa, nos parece que para esses autores há um senso comum na compreensão do termo em questão, não havendo necessidade de conceituar a narrativa. Gomes ${ }^{5}$ 
refere que o termo narrativa, algumas vezes, tem sido utilizado como sinônimo de "depoimentos" ou "discursos", e outras vezes a expressão "narrativas" é empregada sem ter um embasamento teórico, de forma a responder a um modismo. Nos fica o questionamento de qual seria o ganho no uso indiscriminado do termo narrativa. Uma hipótese seria responder a uma tendência das pesquisas qualitativas, que - como já foi mencionado em nossa introdução - valorizam a subjetividade da saúde e da doença, estabelecendo o contraponto entre a experiência sujeito e o enfoque macroestrutural ${ }^{7}$.

Quanto aos sentidos atribuídos ao uso da narrativa nos estudos, observa-se o uso em vários estudos da narrativa como uma técnica para obter depoimentos, Castellanos ${ }^{3}$ menciona que no início "a antropologia e a sociologia utilizavam as narrativas de maneira instrumental"(p.1067), como uma forma de acessar a realidade objetiva, e aponta que atualmente ainda há o uso dessa estratégia. Há de se considerar que no início da antropologia e sociologia ainda havia o comprometimento com a ideia positivista de ciência, valorizando como conhecimento verdadeiro apenas o racionalmente orientado, colocando a narrativa como um mero depoimento a ser colhido.

Entretanto, como citado anteriormente, nota-se que, em geral, o depoimento acerca do adoecimento que se espera obter, está focado na história do sujeito. Nesse sentido, Canesqui ${ }^{7}$ aponta que uma diferença entre a análise narrativa e outras formas de pesquisa qualitativa está na forma da construção da história pelas pessoas, onde há uma "interpretação no contexto que foram geradas, contadas e organizadas no tempo e no espaço"(p. 34).

Essa ideia que a narrativa se configura na temporalidade e na espacialidade é assinalada por Good $^{4}$ como um componente essencial da narrativa, onde a história, o enredo e contexto virtual da narração vão sendo determinados de acordo com a sucessão de acontecimentos. Ricouer ${ }^{48}$ refere que "o mundo exibido por qualquer obra narrativa é sempre um mundo temporal”(p.15), ressalta a relação entre o que denomina "tempo humano" e narrativa, onde esse tempo configura-se em "humano" à medida que se articula com a narrativa, ao mesmo tempo que o significado da narrativa apresenta traços dessa "experiência temporal".

Narrativa e experiência encontram-se intimamente relacionadas, Good ${ }^{4}$ assinala que a estratégia narrativa possibilita o relato e a repre- sentação da experiência, não descrevendo apenas a perspectiva atual, mas também projetando as atividades e experiências para o futuro, de tal forma que organiza os desejos e estratégias do narrador, dirigindo para o final esperado ou formas de experiências que se espera atingir. Para ele, "a experiência vivida e atividades sociais têm, pois, uma complexa relação com as histórias que as referem"(p. 255). Isto ocorre à medida que a experiência do outro suscita, em certo grau, experiências nos ouvintes. Havendo nessa relação uma resposta culturalmente construída.

Se fossemos considerar esses referenciais teórico-conceituais, observamos que os sentidos atribuídos ao uso da narrativa por parte dos autores estudados nem sempre se aproximam ao que se entende por esse termo na literatura especializada acerca do assunto. Entre os artigos analisados, os que se destacam com maior alinhamento conceitual a esse referencial mencionado são os seguintes Harden ${ }^{18}$, Haidet et al. ${ }^{26}$, Dailey ${ }^{43}$, Jacobi e MacLeod ${ }^{16}$ e Afonso et al. ${ }^{19}$.

Quanto aos principais resultados atribuídos ao uso da narrativa, destacam-se dois grupos, um diz respeito a experiência do adoecimento possibilitar a interpretação e ressignificação da cronicidade, consoante ao citado por Bury ${ }^{8}$ como sendo a narrativa a possibilidade de reorganização dos recursos individuais, ressignificando sua nova condição. $\mathrm{O}$ outro grupo diz respeito a importância do conhecimento e compreensão do adoecimento na perspectiva do sujeito adoecido.

Esses dois conjuntos se inter-relacionam e tornam relevante considerar a experiência do sujeito adoecido na compreensão do adoecimento crônico. Canesqui ${ }^{7}$ empreendeu pesquisa documental de artigos de revisão sobre os estudos de doenças crônicas, no período de 1980-2005, refere que especificamente ao rever artigos de determinada revista inglesa, estes tratavam de "várias facetas da experiência da enfermidade"(p.23) menciona exemplos de doenças crônicas e como os portadores lidam com a incerteza e adaptações de convívio com a doença.

A autora ainda cita que as narrativas sobre as enfermidades possibilitaram "novas abordagens sociológicas e antropológicas, que permitem a compreensão do outro, por meio dos seus projetos, manejos de suas vidas, respostas evocadas para o próprio eu"7 (p.34), e que a "recuperação das narrativas rompe com a centralidade do modelo biomédico no discurso da doença"7 (p.34).

Para Bury ${ }^{2}$ o estudo das narrativas de doenças crônicas traz a experiência interrompida, significados e ações tomadas para lidar com essa ex- 
periência, além de trazer o potencial de revelar um conjunto mais vasto de questões importantes para fazer links entre identidade, experiências e culturas da "modernidade tardia".

Bury ${ }^{1}$ menciona a importância das narrativas da doença na história da medicina, fazendo uma clara distinção entre o período pré e pós advento da modelo biomédico. Com o avanço científico da biomedicina, a importância da experiência do paciente e sua narrativa foram diminuídas. Entretanto, com o advento das doenças crônicas, a visão subjetiva do paciente tornou-se audível. Questões como a gestão diária das doenças, avaliação da qualidade de vida, impacto da doença sobre os cuidadores e renegociação do papel dos cuidados profissionais fornecem espaço para as narrativas leigas.

Em contrapartida, há de se considerar um dos estudos, $\mathrm{Miczo}^{17}$ mencionado anteriormente, que explora a possibilidade de ao se dar voz ao sujeito adoecido, a fim de obter sua experiência, existe a possibilidade da manipulação da linguagem através da narração seletiva de histórias.

Assim como referido por Hyden ${ }^{6}$ "narrativas da doença podem ser encontradas em uma variedade de contextos sociais, determinar de diferentes maneiras quem narra, para quem e com que propósitos"(p.62). Torna-se evidente a importância de se considerar o contexto em que ocorre a narrativa.

\section{Considerações Finais}

Os resultados mostraram multiplicidade de sentido atribuídos à narrativa e quanto ao seu uso. Em geral, o termo foi atribuído a uma forma de obter a narração de histórias de vida dos pacientes num determinado tempo e contexto. No que se refere ao seu uso, está intrinsicamente ligado ao sentido atribuído à narrativa, na perspectiva de obter através de entrevistas "abertas" informações a respeito da experiência do adoecimento.

Nos estudos foi pouco observado o uso da narrativa como uma simples escuta, sem uma intencionalidade em seu uso. Uma parte dos estudos não explicitou o sentido ou o uso da narrativa, logo não foi possível a análise, causando lacunas na produção do conhecimento.

Um aspecto importante são os resultados atribuídos ao uso da narrativa nos estudos, em geral os resultados apontaram as experiências dos sujeitos no adoecimento crônico, a percepção, o significado, as estratégias para conviver e enfrentar essa condição, no seu cotidiano e nas relações sociais.

Destacam-se dois principais resultados atribuídos, um diz respeito a possibilidade dos profissionais, por meio da narrativa, compreender o adoecimento crônico a partir da experiência do doente. O outro é a possibilidade de o sujeito da doença ouvir a si próprio e, através da reflexividade, ressignificar o adoecimento crônico e o modo de viver para além da doença.

A possibilidade dessa compreensão ocorre com o uso da narrativa, dessa forma os estudos revelam existir potencial das narrativas em dar sentido às histórias de vida dos sujeitos acometidos por doenças crônicas, principalmente na ressignificação da sua própria condição, e apontar estratégias no cuidado e tratamento no adoecimento crônico.

Em relação aos profissionais de saúde, observamos que, ao explorarem narrativas, podem não só levar em conta a história natural das doenças, mas também contemplarem a temporalidade da experiência dos sujeitos das doenças, levando-os à emersão de racionalidades que possibilitem ressignificar suas ações para lidar com o imenso universo de cenários, personagens e enredos decorrentes do sofrimento do adoecimento crônico ou criados para a superação desse sofrimento.

Um limite desse estudo está na busca das palavras-chave. Verifica-se estudos não contemplados nessa revisão, em virtude da não utilização das palavras-chave doença crônica e narrativa, apontando a importância na escolha das palavras pelos autores, contribuindo para identificação e caracterização da informação existente nos estudos.

Ainda relativo à busca das produções, há outro limite quanto ao fato de que muitos periódicos não atendem aos critérios necessários para indexação em determinadas bases de dados.

Por fim, observa-se que é um tema ainda a ser aprofundado, sugerindo que mais estudos trarão reflexão e importantes contribuições no campo da Saúde Coletiva. Nesse aprofundamento, chamamos atenção para que os novos estudos não só explorem os conteúdos que emergem das narrativas de doenças, mas invistam também na narratividade da doença, ou seja nos estilos como são narradas as experiências, bem como na narração que, em si, já pode ser uma forma de desfantasmagorizar, ressignificar ou gerenciar a doença. 


\section{Colaboradores}

TGV Caçador trabalhou na elaboração do projeto, desenvolvimento da pesquisa, análise de dados e redação do artigo. R Gomes orientou todo processo da pesquisa e participou da revisão da análise e redação do artigo.

\section{Referências}

1. Moreira MCN, Albernaz LV, Sá MRC, Correia RF, Tanabe RF. Recomendações para uma linha de cuidados para crianças e adolescentes com condições crônicas complexas de saúde. Cad Saúde Pública 2017; 33(11):e00189516.

2. Bury M. Illiness narratives: fact or fiction. Sociol Health Illn 2001; 23(3):263-285.

3. Castellanos MEP. A narrativa nas pesquisas qualitativas em saúde. Cien Saude Colet 2014; 19(4):10651076.

4. Good BJ. Medicina, racionalidade y experiencia: uma perspectiva antropológica. Barcelona: Bellaterra; 2013.

5. Gomes R. Pesquisa qualitativa em saúde. São Paulo: Instituto Sírio-Libanês de Ensino e Pesquisa; 2014.

6. Hyden LC. Illness and narrative. Sociol Health Illn 1997; 19(1):48-69.

7. Canesqui AM. Estudos antropológicos sobre os adoecidos crônicos. In: Canesqui AM. Olhares socioantropológicos sobre os adoecidos crônicos. São Paulo: Hucitec; 2007. p.19-51.

8. Bury M. Doença crônica como ruptura biográfica. Tempus Actas Saúde Colet 2011; 5(2):41-55.

9. Castellanos MEP. Cronicidade: questões e conceitos formulados pelos estudos qualitativos de ciências sociais em saúde. In: Castellanos MEP, Trad LAB, Jorge MSB, Leitão IMTA, organizadores. Cronicidade: experiência de adoecimento e cuidado sob a ótica das ciências sociais. Fortaleza: EdUECE; 2015. p.35-60.

10. Greenhalgh T, Hurwitz B. Why study narrative? BMJ 1999; 318(7175):48-50.

11. Kleinman A. The illness narratives: suffering, healing, and the human condition. New York: Basic Books; 1988.

12. Lima TCS, Mioto RCT. Procedimentos metodológicos na construção do conhecimento científico: a pesquisa bibliográfica. Rev Katálysis 2007; 10:37-45.

13. Centro Latino-Americano e do Caribe de Informação em Ciências da Saúde. Portal Regional da BVS: informação e conhecimento para a saúde [Internet]. [acessado 2017 Abr 17]. Disponível em: http://bvsalud.org/

14. Allert G, Sponholz G, Baitsch H. Chronic disease and the meaning of old age. Hastings Cent Rep 1994; 24(5):11-13.

15. Scanlan C, Kerridge I. Autonomy and chronic illness: not two components but many. Am J Bioeth 2009; $9(2): 40-42$.

16. Jacobi S, MacLeod R. Making sense of chronic illness: a therapeutic approach. J Prim Health Care 2011; 3(2):136-141.

17. Miczo N. Beyond the "fetishism of words": considerations on the use of the interview to gather chronic illness narratives. Qual Health Res 2003; 13(4):469-490.

18. Harden J. Parenting a young person with mental health problems: temporal disruption and reconstruction. Sociol Health Illn 2005; 27(3):351-371.

19. Afonso S, Mitre R, Gomes R. Narrativas da experiência de pais de crianças com fibrose cística. Interface (Botucatu) 2015; 19(55):1077-1088.

20. Dekker W, Uerz I, Wils JP. Living well with end stage renal disease: patients' narratives interrupted from a virtue perspective. Ethical Theory Moral Pract 2005; 8(5):485-506. 
21. Faircloth C, Boylstein C, Rittman M, Gubrium J. Constructing the stroke: sudden-onset narratives of stroke survivors. Qual Health Res 2005; 15(7):928941.

22. Docherty D, McColl M. llness stories: themes emerging through narrative. Soc Work Health Care 2003; 37(1):19-39.

23. Erdner A, Andersson L, Magnusson A, Lützén K. Varying views of life among people with long-term mental illness. J Psychiatr Ment Health Nurs 2009; 16(1):54-60

24. Risk J. Building a new life: a chaplain's theory based case study of chronic illness. J Health Care Chaplain 2013; 19(3):81-98

25. Sacks A. Plataforma PyDEsalud: experiencias de pacientes, información, apoyo y capacitación en salud. Med Clin (Barc) 2013; 140(12):544-545.

26. Haidet P, Kroll T, Sharf B. The complexity of patient participation: lessons learned from patients' illness narratives. Patient Educ Couns 2006; 62(3):323-329.

27. Steihaug S, Malterud K. Stories about bodies: a narrative study on self-understanding and chronic pain. Scand J Prim Health Care 2008; 26(3):188-192.

28. Carvalho E, Paiva M, Aparício E, Rodrigues G. Trajetórias afetivo-sexuais de pessoas com feridas crônicas nos membros inferiores: aspectos na escuta terapêutica. Rev Gaucha Enferm 2013; 34(3):163-170.

29. Ayres L, Kavanaugh K, Knafl K. Within-case and across-case approaches to qualitative data analysis. Qual Health Res 2003; 13(6):871-883.

30. Ironside PM, Scheckel M, Wessels C, Bailey ME, Powers S, Seeley DK. Experiencing chronic illness: cocreating new understandings. Qual Health Res 2003; 13(2):171-183.

31. Smith A. Intimacy and family relationships of women with chronic pain. Pain Manag Nurs 2003; 4(3):134142.

32. Hassling L, Nordfeldt S, Eriksson H, Timpka T. Use of cultural probes for representation of chronic disease experience: exploration of an innovative method for design of supportive technologies. Technol Health Care 2005; 13(2):87-95.

33. Ong B, Evans D, Bartlam A. A patient's journey with myalgic encephalomyelitis. BMJ 2005; 330(7492):648650.

34. Harvey I. Self-management of a chronic illness: An exploratory study on the role of spirituality among older African American women. J Women Aging 2006; 18(3):75-88.

35. Angström-Brännström C, Norberg A, Jansson L. Narratives of children with chronic illness about being comforted. J Pediatr Nurs 2008; 23(4):310-316.

36. Schattner E, Shahar G, Abu-Shakra M. 'I used to dream of lupus as some sort of creature': Chronic illness as an internal object. Am J Orthopsychiatry 2008; 78(4):466-472.
37. Washington O, Moxley D, Garriott L, Weinberger J. Five dimensions of faith and spiritually of older African American women transitioning out of homelessness. J Relig Health 2009; 48(4):431-444.

38. Crocker S. Penetrating the dark silence. ANS Adv Nurs Sci 2009; 32(4):295-306.

39. Lee A, Poole G. An application of the transactional model to the analysis of chronic illness narratives. Qual Health Res 2005; 15(3):346-364.

40. Eriksson M, Svedlund M. 'The intruder': spouses' narratives about life with a chronically ill partner. J Clin Nurs 2006; 15(3):324-333

41. Dow C, Roche P, Ziebland S. Talk of frustration in the narratives of people with chronic pain. Chronic Illn 2012; 8(3):176-191.

42. Cross V, Shah P, Glynn M, Chidrawar S. Research into glaucoma and ethnicity (ReGAE) 4: trabeculectomy for advanced glaucoma: the surgical journey of African-Caribbean patients. Eye (Lond) 2008; 22(12):1456-1458.

43. Dailey M. Needing to be normal: the lived experience of chronically ill nursing students. Int J Nurs Educ Scholarsh 2010; 7:Article 15.

44. Naidoo R, Williams $\mathrm{H}$. Therapeutic use of stories for children with atopic eczema and other chronic skin conditions. Pediatr Dermatol 2013; 30(6):765-767.

45. Frank A. Five dramas of illness. Perspect Biol Med 2007; 50(3):379-394.

46. Lucius-Hoene G, Thiele U, Breuning M, Haug S. Doctors' voices in patients' narratives: coping with emotions in storytelling. Chronic Illn 2012; 8(3):163-175.

47. Tropea S. 'Therapeutic emplotment': a new paradigm to explore the interaction between nurses and patients with a long-term illness. J Adv Nurs 2012; 68(4):939947.

48. Ricouer P. Tempo e narrativa. Tomo 1. Campinas: Papirus; 1994.

49. Sanders C, Rogers A, Gardner C, Kennedy A. Managing 'difficult emotions' and family life: exploring insights and social support within online self-management training. Chronic Illn 2011; 7(2):134-146.

Artigo apresentado em 28/08/2018

Aprovado em 05/12/2018

Versão final apresentada em 07/12/2018 